\title{
Intraoperative angiography through the occipital artery and muscular branch of the vertebral artery: Technical Note
}

\author{
Tetsuyoshi Horiuchi, M.D., Junpei Nitta, M.D., Takashi Uehara, M.D., \\ Yuichiro Tanaka, M.D., and Kazuhiro Hongo, M.D. \\ Department of Neurosurgery, \\ Shinshu University School of Medicine, \\ Matsumoto, Japan
}

\author{
Correspondence to: \\ Tetsuyoshi Horiuchi, M.D. \\ Department of Neurosurgery, \\ Shinshu University School of Medicine, \\ 3-1-1 Asahi, Matsumoto 390-8621, Japan \\ Telephone: 81-263-37-2690 \\ Fax: 81-263-37-0480 \\ E-mail: thoriuchi-nsu@umin.ac.jp
}




\begin{abstract}
Background: Intraoperative cerebral angiography has been known as a useful tool for diagnosing neurovascular diseases. There are few reports of the intraoperative angiography in the prone position. Intraoperative angiograms through the occipital artery and muscular branch of the vertebral artery have not also previously been described.

Methods: The occipital artery or muscular branch of the vertebral artery were used for the intraoperative cerebral angiography. With retrograde catheterization of these arteries, the contrast medium (5 to $10 \mathrm{ml}$ ) was manually injected by a surgeon.

Results: Adequate angiogram was obtained with this method. No complications occurred with this procedure.

Conclusions: Intraoperative angiography through these arteries is useful for a patient in the prone position with good efficacy and safety.
\end{abstract}

Key words: Intraoperative angiography, occipital artery, prone position 


\section{Introduction}

Intraoperative angiography has been widely used during neurovascular procedures $(1 ; 10)$. Femoral, radial or retrograde catheterization of the superficial temporal artery have been reported for the intraoperative angiography $(1 ; 4 ; 6 ; 10)$. To the best of our knowledge, there is no previous article describing a use of the occipital artery and muscular branch of the vertebral artery for the intraoperative angiography. The purpose of this article is to describe a new method for the intraoperative angiography through these arteries with a patient in the prone position.

\section{Methods}

Patients: Intraoperative cerebral angiography in the prone position was performed in two patients with a dural arteriovenous fistula (AVF).

Preparation of Patients and Angiography Technique: The patient was placed in the prone position and the head was fixed in the Sugita multipurpose head frame. Before main surgical procedures, the occipital artery or muscular branch of the vertebral artery was identified and isolated (2;9). A J-shaped skin incision was made (Figure 1A) and the musculature was reflected laterally to expose the occipit. The occipital artery was found between the splenius capitis and semispinal capitis muscles (2). Multiple muscular branches originated from the extracranial segments of the vertebral artery. The size, course, and origin of these arteries varied in individual. Since preoperative vertebral angiogram demonstrates the developed muscular branch of the vertebral artery, the branch can be isolated (Figure 2). The artery was catheterized with a 20-gauge intravenous catheter (Figure $1 \mathrm{~B}$ and C). An approximately 20 -mm catheter was inserted in the artery. The catheter was connected to an extension set filled with heparinized normal saline solution (5000 units of heparin in $500 \mathrm{ml}$ ). The continuous heparinized saline was infused through the catheter. The catheter was sutured to the adjacent tissue for fixation (Figure 1B and C). After preparation of the intraoperative carotid angiography, direct surgical isolation of dural sinus was carried out. Brain retractors and associated non-radiolucent equipments were removed, and the operating microscope was withdrawn from the operative field. The craniotomy site was covered with sterile towels and drapes, and the fluoroscopic C-arm (TR-100P-2, Toshiba, Japan) was brought in and positioned. Angiography was performed with manual injection of 5 to $10 \mathrm{ml}$ of contrast medium by a surgeon (Figure 2 and 4). The catheter was withdrawn and the cannulated artery was ligated after completion of angiography.

Illustrative case: This 71-year-old woman was admitted with speech disturbance. Computed tomography showed a subcoritical hematoma at the left temporal lobe. The left carotid injection revealed the sigmoid dural AVF (Figure 3A). Since a transvenous embolization could not succeed, the direct surgery was carried out (the occipital and suboccipital craniotomies with mastoid osteotomy and the isolation of the isolated dural sinus) after the transarterial embolization of the occipital artery. Intraoperative angiograms were obtained before and after the isolation of the sinus (Figure 4). Postoperative angiograms demonstrated the remission of the dural AVF (Figure 3B) and the patient was transferred for rehabilitation. The postoperative angiograms were consistent with the intraoperative one.

\section{Results}

Intraoperative angiography with the occipital artery and muscular branch of the vertebral artery provided sufficient images. Angiographic findings of postoperative angiograms were consistent with those of intraoperative angiograms. No complications related to the intraoperative angiography have occurred. 


\section{Discussion}

The present study has demonstrated that the intraoperative angiography through the occipital artery and muscular branch of the vertebral artery is safe and valuable. Several methods have been proposed for intraoperative angiography and were divided into two groups: catheter method through the femoral or radial artery, and direct cannulation method through the superficial temporal or common carotid arteries.

The standard intraoperative angiography for cerebrovascular surgeries carried out via the transfemoral route. Chiang and colleagues (1) observed eight complications of the 303 operations (2.6\%) related to the femoral approach. Complications of intraoperative angiography included embolism, ischemic stroke, femoral artery thrombosis, pseudoaneurysm formation, arterial dissection, retroperitoneal hemorrhage, broken guidewire, and hematoma formation $(1 ; 3)$. Based on previous reports $(1 ; 3 ; 10)$, the postoperative stroke related to the intraoperative transfemoral angiography ranged from 0 to $0.4 \%$ of patients.

Recently, intraoperative angiography through the radial artery has been introduced and transradial approach has some advantages, especially in the prone position (4). The advantages include continuous access to the sheath during the surgical procedure and easy catheterization of vertebral arteries. In the report of the intraoperative transradial angiography (4), one complication (brachial artery dissection) of 8 patients occurred and the patient received a bypass surgery.

On the other hand, a direct cannulation method using the superficial temporal artery and the common carotid artery was also used to perform the intraoperative angiography $(5 ; 7 ; 8)$. Lee and Macdonald (6) recently represented the efficacy of intraoperative angiography with the superficial temporal artery. Weiner et al.(11) presented one possible cerebral ischemic event among 65 diagnostic angiography through the superficial temporal artery. According to the report by Smith (8), there was one patient among 41 who experienced distal embolization into the internal carotid artery. These ischemic complications would be attributed to placement of the catheter in the common carotid artery or carotid bifurcation (6). Therefore, Lee and Macdonald (6) used a short catheter to avoid such complication. In the present technique, we also used a short catheter and there was no complication related to the intraoperative angiography.

CONCLUSIONS: Intraoperative angiography via the occipital artery and muscular branch of the vertebral artery is a feasible alternative to transfemoral or transradial catheterization in the prone position. In the present report, adequate angiograms can easily be obtained without complications. To the best of our knowledge, this is the first report describing the use of the occipital artery and muscular branch of the vertebral artery to obtain the intraoperative angiogram for neurovascular surgery. 


\section{Figure legends}

Figure 1. A: Illustration of the skin incision. B: Intraoperative photograph of catheterization of the left occipital artery. C: Magnified view of the catheterization

Figure 2. A: Preoperative vertebral angiogram showing the developed muscular branch (MB). B: Intraoperative angiography of the vertebral artery through the MB.

Figure 3. Preoperative (A) and postoperative (B) left carotid angiograms. Note that the dural arteriovenous fistula disappears after surgery.

Figure 4. Intraoperative angiography of the internal carotid artery through the occipital artery (OA) before (A) and after (B) the isolation of the sinus. Note that cortical refluxes remit after the isolation. 


\section{Reference List}

1. Chiang VL, Gailloud P, Murphy KJ, Rigamonti D, Tamargo RJ. Routine intraoperative angiography during aneurysm surgery. J Neurosurg 96:988-992, 2002.

2. Horiuchi T, Nitta J, Kitazawa K, Nagashima H, Iwashita T, Koyama J, Uehara T, Tanaka Y, Hongo K. Direct surgical treatment with OA-PICA anastomosis for ruptured PICA-involved VA dissecting aneurysm. Surg Cereb Stroke 33:402-405, 2005.

3. Klopfenstein JD, Spetzler RF, Kim LJ, Feiz-Erfan I, Han PP, Zabramski JM, Porter RW, Albuquerque FC, McDougall CG, Fiorella DJ. Comparison of routine and selective use of intraoperative angiography during aneurysm surgery: a prospective assessment. $\mathbf{J}$ Neurosurg 100:230-235, 2004.

4. Lang SS, Eskioglu E, Mericle A. Intraoperative angiography for neurovascular disease in the prone or three-quarter prone position. Surg Neurol 65:283-289, 2006.

5. Lazar ML, Watts CC, Kilgore B, Clark K. Cerebral angiography during operation for intracranial aneurysms and arteriovenous malformations. Technical note. J Neurosurg 34:706-708, 1971.

6. Lee MC, Macdonald RL. Intraoperative cerebral angiography: superficial temporal artery method and results. Neurosurgery 53:1067-1074, 2003.

7. Parkinson D, Legal J, Holloway AF, Walton RJ, Lafrance RR, MacEwan DW, Johnson J. A new combined neurosurgical headholder and cassette changer for intraoperative serial angiography. Technical note. J Neurosurg 48:1038-1041, 1978.

8. Smith RW. Intraoperative intracranial angiography. Neurosurgery 1:107-110, 1977.

9. Sundt TM, Jr., Piepgras DG. Occipital to posterior inferior cerebellar artery bypass surgery. J Neurosurg 48:916-928, 1978.

10. Tang G, Cawley CM, Dion JE, Barrow DL. Intraoperative angiography during aneurysm surgery: a prospective evaluation of efficacy. J Neurosurg 96:993-999, 2002.

11. Weiner IH, Azzato NM, Mendelsohn RA. Cerebral angiography; a new technique; catheterization of the common carotid artery via the superficial temporal artery. $\mathbf{J}$ Neurosurg 15:618-626, 1958. 